\title{
BMJ Open Link workers providing social prescribing and health and social care coordination for people with multimorbidity in socially deprived areas (the LinkMM trial): protocol for a pragmatic randomised controlled trial
}

\author{
Bridget Kiely, ${ }^{1}$ Barbara Clyne (D) , ${ }^{1}$ Fiona Boland (D) , ${ }^{1}$ Patrick O'Donnell, ${ }^{2}$ \\ Deirdre Connolly, ${ }^{3}$ Eamon O'Shea, ${ }^{4}$ Susan M Smith (D) ${ }^{1}$
}

To cite: Kiely B, Clyne B, Boland $\mathrm{F}$, et al. Link workers providing social prescribing and health and social care coordination for people with multimorbidity in socially deprived areas (the LinkMM trial): protocol for a pragmatic randomised controlled trial. BMJ Open 2021;11:e041809. doi:10.1136/ bmjopen-2020-041809

- Prepublication history for this paper is available online. To view these files, please visit the journal online (http://dx.doi. org/10.1136/bmjopen-2020041809).

Received 17 June 2020 Revised 01 December 2020 Accepted 07 December 2020

Check for updates

(c) Author(s) (or their employer(s)) 2021. Re-use permitted under CC BY-NC. No commercial re-use. See rights and permissions. Published by BMJ.

For numbered affiliations see end of article.

Correspondence to

Dr Bridget Kiely;

bridgetkiely@rcsi.com

\section{ABSTRACT}

Introduction Link workers are non-health or social care professionals based in primary care who support people to develop and achieve a personalised set of health and social goals by engaging with community resources. Link workers have been piloted in areas of deprivation, but there remains insufficient evidence to support their effectiveness. Multimorbidity is increasing in prevalence, but there are limited evidence-based interventions. This paper presents the protocol for a randomised controlled trial (RCT) that will test the effectiveness of link workers based in general practices in deprived areas in improving health outcomes for people with multimorbidity.

\section{Methods and analysis The protocol presents} the proposed pragmatic RCT, involving 10 general practitioner (GP) practices and 600 patients. Eligible participants will be community dwelling adults with multimorbidity ( $\geq$ two chronic conditions) identified as being suitable for referral to a practice-based link worker. Following baseline data collection, the patients will be randomised into intervention group that will meet the link worker over a1-month period, or a 'wait list' control that will receive usual GP care. Primary outcomes are health-related quality of life as assessed by EQ-5D-5L and mental health assessed by Hospital Anxiety and Depression Scale. Secondary outcomes are based on the core outcome set for multimorbidity. Data will be collected at baseline and on intervention completion at 1 month using questionnaires selfcompleted by participants and GP records. Parallel process and economic analyses will be conducted to explore participants' experiences and examine costeffectiveness of the link worker intervention.

Ethics and dissemination Ethical approval has been granted by the Irish College of General Practitioners Ethics Committee. The findings will be published in peerreviewed journals.

Trial registration number ISRCTN10287737; Pre-results.
Strengths and limitations of this study

- The LinkMM study is a pragmatic randomised controlled trial examining the effectiveness of a practice-based link worker intervention for people with multimorbidity.

- The focus on people with multimorbidity builds the evidence base for generic interventions that work across all conditions in multimorbidity.

- The short intervention and follow-up period allows for a wait list control design and is consistent with the duration of real world link worker interventions, but may be too short to show a meaningful difference in outcomes.

- There is large number of patient-reported outcomes, consistent with the Medical Research Council guidance on evaluating complex interventions, but this may be off-putting to people with lower literacy levels, creating challenges for recruitment and potential threats to generalisability for very vulnerable adults with multimorbidity.

- Parallel process and economic analysis will add to our understanding of the implementation of this type of intervention and determine the cost-effectiveness of the intervention.

\section{INTRODUCTION}

Multimorbidity, defined as the presence of two or more chronic conditions, is recognised as a significant challenge for patients and healthcare systems, particularly in primary care and in areas of social deprivation. ${ }^{1}$ Within the broader multimorbidity population, there are people with higher numbers of conditions involving multiple body systems with related polypharmacy, which is referred to as complex multimorbidity. ${ }^{2}$ Multimorbidity and complex multimorbidity are estimated to affect $66.2 \%$ and $11 \%$, respectively, 
of people over 50 years of age attending Irish General Practice. ${ }^{3}$ Complex multimorbidity is associated with increased healthcare utilisation and costs. People with complex multimorbidity experience fragmented care, poorer mental health and have worse outcomes. ${ }^{45}$ There are higher proportions of patients with complex combinations of physical and mental health conditions in deprived areas. ${ }^{4}$ This is reflected in higher consultation rates and has ramifications throughout the health system. A total of $10 \%$ of patients with 4 or more conditions account for $34 \%$ of unplanned emergency admissions and $47 \%$ of preventable unplanned admissions. ${ }^{6}$ People living in deprived areas develop multimorbidity 11 years earlier ${ }^{4}$ and experience worse quality of life compared with those with multimorbidity in less deprived areas. ${ }^{7}$ It is not clear why this is, but there is growing evidence that people with multimorbidity in areas of deprivation have reduced selfefficacy and capacity for self-management due to psychosocial stressors, poorer mental health, increased burden of treatment and lower perceived social support. ${ }^{8-13}$

There is as yet, limited evidence to indicate which interventions for multimorbidity have a significant impact on health outcomes or health service utilisation. ${ }^{14}$ One potential intervention to address the complex mix of psychosocial issues and multimorbidity in areas of deprivation is the use of link workers in primary care. A link worker is a non-health or social care professional who usually has training in coaching or behaviour change, as well as an extensive knowledge of local community resources. They work with people referred to them by healthcare services to identify their health and social care needs, and support them to access services within the community to improve their health and well-being, a process commonly referred to as social prescribing. ${ }^{15}$ The Glasgow Deep End Links Worker programme describes the principle behind the link worker intervention as 'a catalyst to hope and self-determination, using the strong relationships with patients that exist in general practice. If patients with complex needs feel supported, they would be more likely to respond to information on ways to improve their health'. The current study builds on this work using a similar intervention approach with link workers embedded in practices in deprived urban areas.

Although link workers providing social prescribing have been gaining popularity in the UK and there have been a number of pilots in Ireland, ${ }^{16}{ }^{17}$ few have been formally evaluated. A recent review of link worker provided social prescribing in the UK found limited evidence to support the effectiveness and concluded that there was a lack of evidence for how, for whom and when social prescribing was effective. ${ }^{18}$ A recent quasi-experimental evaluation of the Glasgow Deep End Links Worker programme found some impact on mental health scores for patients and staff morale in general practitioner (GP) practices and concluded that larger, longer studies with randomisation at the individual patient level were needed. ${ }^{19}$

The Deep End Ireland GP group, a network of practices based in areas of deprivation, prepared a report on link worker provided social prescribing in Ireland that outlined its potential to address the psychosocial burden faced by their patients and the impacts of upstream social determinants of heath that GPs often encounter, but can have little impact on in practice. ${ }^{20}$

To inform the implementation of the intervention and evaluation processes a short uncontrolled pilot study was conducted in one practice with 12 patients. This confirmed the feasibility of intervention delivery and led to refinements in patient selection and data collection processes (paper in submission process with the Journal of Comorbidity).

This study aims to evaluate a link worker intervention in primary care on health outcomes for people with complex multimorbidity in socially deprived areas. Secondary aims are to examine the impact on staff morale and conduct a mixed methods process evaluation and economic evaluation of the intervention, exploring direct and indirect costs.

\section{METHODS}

This protocol is presented using the Standard Protocol Items: Recommendations for Interventional Trials recommendations for the reporting of a protocol for an interventional trial. ${ }^{21}$

\section{Study design}

This will be a pragmatic randomised controlled trial (RCT) to evaluate a link worker intervention in improving health outcomes for people with multimorbidity attending primary care in socially deprived areas compared with wait list controls who receive usual care. It will be reported in accordance with the Consolidated Standards of Reporting Trials guidance for RCTs. ${ }^{22}$ The economic analysis will be a cost utility analysis from the perspective of the public healthcare system and will be carried out in accordance with the guidance produced by the Health and Information Quality Authority Ireland. ${ }^{23}$

A parallel mixed methods process evaluation will be conducted in line with the Medical Research Council (MRC) guidance on evaluating complex interventions. ${ }^{24}$ The process evaluation protocol will be informed by the MRC framework for process evaluations. ${ }^{25} \mathrm{~A}$ full protocol for the process evaluation will be published in an open access source.

\section{Public and patient involvement}

This study has public and patient involvement (PPI) through a multimorbidity patient advisory group. The patient advisory group are patients with multimorbidity who meet quarterly to discuss issues arising with research projects on multimorbidity funded through the Health Research Board Collaborative Doctoral Award (BK is a PhD student on this programme). The specific input of the PPI groups is outlined in the pilot study paper (paper in submission process with the Journal of Comorbidity) but, in summary, it included co-design of patient 
information leaflets, input on patient outcome selection and questionnaire design.

\section{Implementation advisory group}

The implementation advisory group consists of GPs working in deprived areas, with and without experience of social prescribing and a project manager from a wellestablished social prescribing project in a deprived inner city area in Dublin, Ireland.

\section{Study settings}

This study will be conducted in urban general practices serving areas of deprivation in four cities (Dublin, Cork, Limerick and Waterford) in Ireland. Serving areas of deprivation will be defined as providing general practice care to at least two small areas identified as disadvantaged or below by the Pobal HP Deprivation Index and providing services to at least 1000 patients under the General Medical Services (GMS) scheme. The Pobal HP Deprivation Index is Ireland's most widely used social gradient metric and scores each small area (50-200 households) in terms of affluence or disadvantage. The index uses information from Ireland's census, such as employment, age profile and educational attainment, to calculate this score. ${ }^{26}$ The GMS scheme provides medical care to approximately $40 \%$ of the Irish population. It is predominantly means-tested and provides eligible patients with free GP visits, free hospital care and free medications (except for a prescription levy, currently $€ 2.50$ for every item to a maximum of $€ 25$ ).

\section{Eligibility criteria}

Participants

Participants will be community dwelling adults aged over 18 , who have two or more chronic conditions (multimorbidity), attend a GP who provides care for patients living in an area of deprivation and have been identified by their GP as having potential to benefit from a link worker intervention. As this is a pragmatic trial, we are seeking to replicate conditions in real work practice where GPs would refer to a link worker based on need. There will be no predefined conditions, other than the conditions should be chronic, that is lasting or expected to last more than 6 months.

Exclusion criteria include psychiatric/psychological morbidity or cognitive impairment that would impair capacity for informed consent, a terminal illness likely to lead to death or major disability during the study follow-up period, living in residential care, already participating in a similar programme or had previously participated in the pilot study.

\section{Practices}

Ten practices in the Deep End Ireland group will be invited to participate. ${ }^{27}$ Membership of the group is open to any practice that identifies as working in an area of deprivation. In addition, practices must have a GMS list of $>1000$ patients, serve at least two small areas defined as disadvantaged or below by the Pobal HP Deprivation
Index 2016 and have space to host a link worker on site. Practices that are taking part in another link worker project will be excluded.

\section{Recruitment and randomisation}

Each practice will be asked to recruit 60 participants, giving a total of 600 patients. Recruitment will begin 1 month before the start date of the intervention and will be phased for logistical reasons with 20 participants being recruited each month in each practice.

Eligible participants will be identified by their GPs, based on being prescribed five or more medications as a proxy for multimorbidity. This proxy is being used because of significant variation in coding practices for chronic conditions in Ireland and lack of a code for multimorbidity. A finder tool in the electronic record, previously developed for another multimorbidity study, ${ }^{28}$ will generate this list of patients. Previous research has indicated that medication count is a suitable proxy measure for multimorbidity. ${ }^{6}$ The GP team will screen this list of patients with multimorbidity to identify all patients who they would refer to a link worker and thus create a register of potentially eligible patients. This process is based on our pilot study findings and is designed to reflect realworld conditions where GPs refer patients they identify as having a psychosocial need that would benefit from a social prescribing approach to a link worker. Once this register of potentially eligible patients is created, GPs will be supported to arrange the list in random order and select the first 30 potential participants to invite. They will then be asked to double check that the selected participants meet the inclusion criteria and ensure none of the exclusion criteria apply. GPs will also be asked to document the reasons why each of the selected patients would be referred to a link worker using a standardised list of options. This list will include reasons for referral identified from other studies and known proxies for psychosocial need, such as frequent attendance and will also allow GPs to record free text additional reasons. This process will improve transparency around referral decision making and provide additional data on types of patients referred for link worker supports and is summarised in figure 1 .

The research team will update the GP practices on a fortnightly basis of who has returned consent forms. The GPs will be encouraged to remind anyone who had verbally agreed to take part during the phone call stage, but not yet returned a consent form to do so. At monthly intervals, the GPs will be asked to invite another 30 patients from the randomly ordered list of potential participants until the total of 60 participants has been reached. Recruitment will end 10 weeks before the end of the study to allow for sufficient time for baseline data collection, randomisation and delivery of the intervention. The expected recruitment rate is $60 \%$ based on the quasi-experimental evaluation of the Glasgow Links Worker project ${ }^{19}$ where $50 \%$ of potential participants were recruited and our own 
- GPs screen this list to identify patients they would refer to a link worker

- GPs put this list in random order and send invitation packs in batches of 30, after
double checking they meet eligibility criteria

Figure 1 Process for selection and recruitment of participants. GP, general practitioner.

pilot where $70 \%$ of invited participants returned consent and baseline questionnaires.

A letter of invitation, patient information leaflet, and consent and baseline questionnaires will be sent to eligible participants and GP teams will follow-up with phone calls to explain the study and see if potential participants require assistance completing the baseline questionnaires. One in six Irish adults are functionally illiterate ${ }^{29}$ and so it is assumed that at least this number will require their GP to verbally explain the study and need assistance with completion of the baseline questionnaires. Once they have consented, a member of the research team can assist them with baseline data collection either face to face at the practice or over the phone.

Randomisation will take place following baseline data collection to avoid allocation bias. Randomisation will be carried out by an independent researcher and overseen by the trial statistician using a computer-generated sequence. Patients will be stratified by practice and age, and allocation will be blocked using random permuted blocks of sizes two and four to ensure balanced numbers of intervention and control patients in each practice.

The independent researcher will inform the research team of allocations. The research team will contact the participants by phone to inform them of their allocation and what to expect. The research team will inform the relevant link worker by phone who has been allocated to the intervention group. A letter will be sent to the GP practice informing them of the participants involvement in the trial and their allocation. Due to the nature of the intervention, it is not possible to blind participants, link workers or GPs to the allocation. Blinding will be implemented at the data analysis stage.

\section{Intervention}

The link worker intervention (LinkMM) is based on the Glasgow Deep End Links Worker project which had a quasi-experimental cluster design. ${ }^{30}$ Our intervention is shorter than the Glasgow project to facilitate the wait list control study design and it does not include practice subsidies for developing in-practice activities beyond hosting the link worker. The LinkMM is a complex intervention with the following components:

- Link worker training and support.

- GP training.

- Compilation and mapping of local health and social care community resources.

- Link worker participant meetings and follow-up.

- Financial supports to practices.

\section{Link worker training and support}

To inform the implementation of link worker social prescribing project, the lead researcher attended Social Prescribing Network Ireland meetings and engaged with local social prescribing projects to explore the nature of the link worker role and appropriate job specifications, training, communication with GPs and engaging with community resource providers. In keeping with the literature, empathy and an ability to listen were identified as important link worker skills. ${ }^{31}$ Given the limited training time available, a background in health or social care and experience of working with disadvantaged communities were essential criteria for applicants to the role.

Resources from the Alliance website ${ }^{32}$ (the community organisation who had delivered the link worker intervention in the Glasgow Deep End Links Worker project) were referenced to develop a 40-hour training plan with input 
from local social prescribing providers and based on the experience of our pilot study link worker, who had significant experience in health and social care, and working with disadvantaged communities. Link workers will be employees of the research host institution rather than the general practices and will be line managed by the trial project manager. They will have monthly check-ins with the project manager and bimonthly peer support meetings and review sessions. Any clinical concerns regarding participants will be raised with the individual's GP. If for whatever reason they are unable to access the individual's GP, link workers can seek support from the principal investigator (SMS), an experienced GP.

\section{GP training}

GP practices will receive training on site or via video link on trial processes including selection and recruitment processes and the potential reasons a patient might be referred to a link worker. The link worker role will be explained.

\section{Compilation and mapping of local health and social care community resources}

The research team will have identified some key local resources for each area in advance of the link worker taking up their post. The link workers will have allocated time during their induction period to map out local resources using a template developed during the pilot study. This will, however, be an ongoing process depending on the needs of participants. In the context of the ongoing COVID-19 pandemic, online and individual resources will also be identified for those unable to attend group activities.

\section{Link worker participants meetings and follow-up}

Following randomisation, intervention group participants will be referred to the link worker straightaway and invited to meet with them at least once, with at least $60 \mathrm{~min}$ scheduled for this initial appointment. At the initial meeting, the link worker will explain their role and explore the participants' health and social care priorities, and produce a joint plan to address these. This will include a range of activities and community resources that participants may choose to attend to improve their health and well-being. The link worker will offer to follow-up and support participants to implement their plan. It is expected that support will broadly fall into one of four categories, informational (supplying information on resources and directing to websites), instrumental (making an appointment on behalf of a participant or accompanying them to an appointment), appraisal (helping participants to makes changes using behaviour change techniques, such as motivational interviewing) or emotional (listening and encouraging when participants face challenges) ${ }^{33}$ As support is tailored to the needs of the individual, it will vary. All link worker activity will be captured in a specifically designed client management database, including details of the initial assessment, priority health and social issues, goals set, community resources referred to and attended, number of follow-ups and the type of support provided, as per the categories outlined above. This will be fully reported in a parallel process evaluation and briefly described in the main trial report. There will be no change to the participants usual clinical care. At the end of the intervention period, the link worker will provide a summary to the participant's GP, outlining the plan and resources they accessed to help achieve it.

The link worker will be based in the GP's practice and meetings with participants will primarily be in the GP's practice. The link worker will be able to liaise with the GP of the participant should they have any specific concerns about an individual. Link workers will also share knowledge on local community resources with GPs during monthly meetings with practice staff.

\section{Financial supports to practices}

Practices will receive a stipend to cover one session a week of GP's time to allow for time spent on recruitment and supporting the link worker intervention. An additional grant will cover any room hire and equipment costs that the practice may incur as a result of hosting the link worker.

\section{Control}

The RCT will have a wait list control. During the 1-month intervention period, the control group will receive usual care from their GP. On completion of the intervention, the control group will be invited to a one-off meeting with the link worker, during which they will have an opportunity to identify their needs and be provided with a list of suggested resources tailored to these.

\section{OUTCOMES}

A wide range of outcomes will be used to assess intervention effectiveness and mechanism of action in line with the MRC framework for evaluating complex interventions. ${ }^{24}$ Outcomes are based on the pilot study findings and on the Core Outcome Set for Multimorbidity research. ${ }^{34}$ In line with the National Institute for Health and Care Excellence, an additional measure of capability and well-being, the ICEpop CAPability measure for Adults (ICE-CAP A) ${ }^{35}$ will be used alongside the EQ-5D-5L to capture the wider social benefits to the individual that are expected with this type of intervention.

\section{Primary outcomes}

- Health-related quality of life (HRQoL) as measured by EQ-5D-5L. ${ }^{36}$

- Mental health as measured by Hospital Anxiety and Depression Scale (HADS). ${ }^{37}$

\section{Secondary outcomes}

Patient-reported outcome measures

- Capability and well-being as measured by the ICE-CAP A. ${ }^{35}$ 
- Activities of daily living as measured by the Frenchay Activities Index. ${ }^{38}$

- Self-management as measured by the Patient Activation Measure. $^{39}$

- Burden of treatment as measured by Multimorbidity Treatment Burden Questionnaire. ${ }^{40}$

\section{Healthcare utilisation}

Data from primary care electronic health records in the previous month unless otherwise specified:

- Number of GP attendances.

- Number of practice nurse attendances.

- Number and type of regularly prescribed medications.

- Number of out-of-hours GP attendances.

- Number of emergency department attendances.

- Number of hospital admissions (emergency) and length of stay.

- Number of hospital outpatient visits.

\section{Sample size}

A sample size of 600 participants in total has been calculated based on our two primary outcomes. Using a HADS Anxiety score of 10.9 , a SD of 5.1 and a minimally clinically important difference of 1.5 (based on a similar Scottish population); ${ }^{30}$ for $90 \%$ power with approximately $20 \%$ loss to follow-up, 600 patients are needed. Similar calculations for HRQoL, using 0.316 SD units in EQ5D (based on a similar Scottish population) ${ }^{30}$, for $90 \%$ power and presuming approximately $20 \%$ loss to follow-up, we need a sample of 510 patients. We will recruit the larger number of 600 patients (300 in each study arm).

\section{Data collection}

Data collection will be at baseline prerandomisation and at 1 month from initial invitation to the intervention group to meet with the link worker. This will be prior to the control group meeting once with the link worker and receiving a truncated version of the intervention.

Patient reported outcome measures will be selfreported using standardised paper-based questionnaires, which will be posted to participants. Data on patient costs and community resources accessed, will be self-reported using a specifically designed questionnaire. A member of the research team will assist participants to fill in paperbased forms if there are literacy issues.

The healthcare utilisation data on GP visits, out-ofhours GP visits, prescribed medications and emergency department attendances will be extracted from healthcare records by a member of the research team.

On completion of the RCT, we also plan to conduct an observational study on all participants at 9 months from the intervention group receiving an invitation to meet with the link worker to examine changes in outcomes over this longer time period.

\section{Data management}

Paper questionnaires will be returned by post to the research team in the Department of General Practice, Royal College of Surgeons in Ireland, who will be responsible for manual data entry into predesigned excel spreadsheets. All participants will provide informed consent for the processing of their data. Data will be pseudonymised with the use of a unique study identification code. All data will be stored in secure encrypted institutional network drives accessible only to named members of the research team. A comprehensive data management plan is in place, which had been reviewed by the trial steering committee (TSC).

A TSC comprising an independent chair and three other independent members, one of whom is a lay member representing the patient and public perspective, has been established and will oversee the progress of the trial and adherence to the study protocol.

Unintended consequences will be monitored during the trial using self-reporting by participants, reporting by link workers during planned supervision and reporting by GPs. GPs will receive instructions on how to report any adverse events on concerns they have to the trial manager during their training on the intervention. In addition, the trial manager will check in on a monthly basis with practices to get updates on any recruitment, implementation challenges and adverse events. Unintended consequences will also be explored as part of the process evaluation.

\section{Planned statistical analysis}

Descriptive statistics will be used to describe recruited participants and to investigate comparability of trial groups at baseline. For primary and secondary outcomes, the primary analyses will be 'intention-to-treat', including all randomised participants, all retained in the group to which they were allocated and using last observation carried forward for missing values. The primary analysis will be adjusted for baseline scores and stratification variables, age and practice. Subsequent models will adjust for multimorbidity severity. We will also conduct a perprotocol (PP) analysis. The PP population will consist of those randomised to the intervention group who met with the link worker at least once. We will also conduct preplanned subgroup analyses based on gender and age (above and below 65 years of age). All analyses will use appropriate (ie, linear or Poisson) regression models with results presented as point estimates (difference in means or incident rate ratios), 95\% CIs and $\mathrm{p}$ values. Stata 15 will be used for all data analysis. ${ }^{41}$

\section{ECONOMIC EVALUATION}

The health economic evaluation will consist of a trialbased cost utility analysis of the proposed intervention. The evaluation will be undertaken in a manner consistent with guidelines issued by the Health Information and Quality Authority in Ireland. ${ }^{23}$ Evidence collected on direct costs of the intervention from the trial, community resource use and health outcome measures will provide the basis for the evaluation over the trial follow-up period. With respect to costing, a publicly funded health service perspective will be adopted. That is, resource use 
associated with delivery of the proposed intervention will be measured and costed, as will other health service resource use by patients over the course of the trial. For the cost utility analysis, effectiveness will be evaluated in terms of quality-adjusted life years (QALYs), which will be estimated based on responses to the EuroQol EQ-5D-5L instrument. ${ }^{35}$

An incremental analysis will be undertaken to provide information on the marginal costs and effects of the intervention relative to the control through the calculation of incremental cost effectiveness ratios (ICERs).

Probabilistic sensitivity analysis will be performed using the range of uncertainties from the statistical analysis of the trial. This allows the expected value of perfect information (EVPI) to be calculated. In this case, given that the data will come from a single trial, this will help to inform whether longer follow-up is worth considering before investing in the intervention.

A cost-effectiveness acceptability curve (CEAC) will be produced to examine the probability of the intervention being cost-effective at different cost-effectiveness thresholds. ICER for every QALY gained will be presented along with a scatter plot, CEAC and EVPI.

\section{PROCESS EVALUATION}

A mixed methods process evaluation is planned and we will publish a separate protocol outlining the methods for this evaluation, to be submitted to HRB Open Research.

\section{DISCUSSION}

This trial will provide some of the most robust results to date on link workers. As a recent systematic review on link worker interventions concluded, there is insufficient evidence 'to judge either success or value for money' and 'future evaluations must be comparative by design and consider when, by whom, for whom, how well and at what cost' interventions are provided. ${ }^{18}$ While previous projects have not specified strict inclusion criteria and have often focused on younger patients with mental health problems, ${ }^{42}$ we are focusing on multimorbidity, which is predicted to increase in prevalence and is known to be a particular challenge in areas of deprivation. This will contribute to evidence on who is most likely to benefit from link workers and social prescribing as well as providing robust effectiveness and economic data.

A recent systematic review of the self-management characteristics of patients with complex health needs concluded that tailored self-management support is required for people in areas of socioeconomic deprivation to address the social norms that accept poorer health, social isolation and socioeconomic insecurity. ${ }^{43}$ Link workers are one intervention that could provide this kind of support. Governments are recognising this and link workers are specifically mentioned in the UK NHS Long Term Plan and funding provided to primary care clinical commissioning groups for one link worker for every 30000 population. ${ }^{44}$ An all-party Committee on the Future of Healthcare in the Irish Parliament agreed on a plan for healthcare reform in Ireland in 2017, called Slaintecare. ${ }^{45}$ This is now being implemented through the Department of Health and Children and it emphasises a shift towards care in the community and empowering people to manage their own health. ${ }^{46}$ Social prescribing is recognised as one way to achieve this. The Department of Health announced a Slaintecare Integration Fund programme in 2019 which is funding a range of projects evaluating interventions that reflect Slaintecare priorities, including this study. Social prescribing is also being supported by the Irish national Health Service Executive (HSE) with a number of funded pilots. ${ }^{16}{ }^{17}$ However, the lack of robust evaluation is recognised and the HSE are in the process of developing an evaluation framework. The results of this trial and process evaluation will be timely in informing national policy about the role-out of link workers and social prescribing nationally.

\section{Strengths}

While there have been a number of smaller trials and quasi-experimental studies, this trial will be the first large scale pragmatic randomised trial of a link worker and social prescribing intervention. This will overcome the previous challenge of finding suitable controls in non-randomised trials ${ }^{19} 47$ and provide some of the most robust results to date on link workers. Furthermore, this will be the first multimorbidity trial with a link worker type intervention and this intervention addresses the challenge of identifying a generic intervention that works across all conditions, as recommended in the Cochrane review of interventions for multimorbidity. ${ }^{14}$

\section{Limitations}

Our study is restricted to deprived urban areas. Rural areas provide unique challenges and have less concentrated deprivation, which would affect costs and recruitment timelines. While deprivation in Ireland is concentrated in urban areas, the results of this trial may not be applicable to more rural locations. Due to the nature of the funding and the wait list control design, our intervention is only 1 month in duration and this may be too short a time to both deliver and show a significant difference in outcomes. While the intervention period is shorter than the Glasgow Deep End Links Worker model, it is in keeping with pilot projects in Ireland where link workers supported people over a $6-8$ week period. ${ }^{42}$ In order to better understand the mechanisms of impact of the intervention, we are collecting a range of measures, but a lengthy questionnaire may be off-putting to potential participants, especially those who are most deprived, leading to a biased sample. The levels of patient engagement in the pilot study, however, are encouraging and the further input from our patient advisory group and expert panel of GPs on recruitment strategies and materials should mitigate against this. The method in which participants are selected may result in a selection bias, 
with GPs selecting participants based on the GPs perception of psychosocial needs. This is, however. the way such an intervention would be implemented in real-world clinical practice, and so the trial is designed to be pragmatic. To better understand why GPs have selected participants, they will be asked to document a reason and this will be reported to provide greater transparency into the selection of participants. Overall, this pragmatic RCT will add to the evidence base for link workers and social prescribing at a time when there is considerable national and international interest in rolling out this intervention more widely.

\section{Ethics approval and dissemination}

Ethical approval has been granted by the Irish College of General Practitioners Ethics Committee. This includes a Data Management Plan and Data Impact Assessment Form to ensure adherence to General Data Protection Regulation and Health Research Regulations.

The end study results will be published in peer-reviewed journals and will be open access. A full report will also be submitted to funders. The results will also be disseminated to relevant stakeholders and participating GP practices. The PPI panel will be consulted on how best to disseminate results to people with multimorbidity.

\section{Author affiliations}

${ }^{1}$ Department of General Practice, HRB Centre for Primary Care Research, Royal College of Surgeons, Dublin, Ireland

${ }^{2}$ Primary Healthcare, Graduate Entry Medical School, University of Limerick, Limerick, Ireland

${ }^{3}$ Discipline of Occupational Therapy, Trinity College, Dublin, Ireland

${ }^{4}$ School of Business and Economics, National University of Ireland, Galway, Ireland

\section{Twitter Bridget Kiely @bridgetmkiely and Susan M Smith @susanmsmithm}

Contributors BK is the lead researcher for the pilot project and randomised controlled trial, completed the initial draft, contributed to manuscript drafts, approved the final draft and will act as corresponding author. BC gave advice on methodology for the pilot and main randomised controlled trial, and contributed to manuscript drafts and approved the final draft. FB is the lead statistician for project, gave advice on randomisation methods and planned statistical analysis. She also contributed to manuscript drafts and approved the final draft. PO'D contributed to manuscript drafts and approved the final draft. DC contributed to manuscript drafts and approved the final draft. EO'S is the lead economist and advised on economic methods and analysis plans. SMS conceptualised the original research question and trial methodology. All authors reviewed and approved the final draft.

Funding This trial is jointly funded by the Health Research Board Ireland (Grant reference HRB CDA 2018 Reference CDA-2018-003) and the Department of Health Slaintecare Integration Fund (Grant reference PCC-ID24). The funders did not have any role in the design of this study.

\section{Competing interests None declared.}

Patient and public involvement Patients and/or the public were involved in the design, or conduct, or reporting or dissemination plans of this research. Refer to the Methods section for further details. Fully informed consent will be obtained from all participants. Confidentiality will be maintained by pseudonymisation of data using a unique study ID. Only named members of the research team will have access to individuals personal contact data and will only access it to communicate with participants regarding the trial. All data will be stored in secure password protected files with named access only.

Patient consent for publication Not required.

Provenance and peer review Not commissioned; externally peer-reviewed.
Open access This is an open access article distributed in accordance with the Creative Commons Attribution Non Commercial (CC BY-NC 4.0) license, which permits others to distribute, remix, adapt, build upon this work non-commercially, and license their derivative works on different terms, provided the original work is properly cited, appropriate credit is given, any changes made indicated, and the use is non-commercial. See: http://creativecommons.org/licenses/by-nc/4.0/.

\section{ORCID iDs}

Barbara Clyne http://orcid.org/0000-0002-1186-9495

Fiona Boland http://orcid.org/0000-0003-3228-0046

Susan M Smith http://orcid.org/0000-0001-6027-2727

\section{REFERENCES}

1 Fortin M, Soubhi H, Hudon C, et al. Multimorbidity's many challenges. BMJ 2007;334:1016-7.

2 Harrison $\mathrm{C}$, Britt $\mathrm{H}$, Miller $\mathrm{G}$, et al. Examining different measures of multimorbidity, using a large prospective cross-sectional study in Australian general practice. BMJ Open 2014;4:e004694.

3 Glynn LG, Valderas JM, Healy P, et al. The prevalence of multimorbidity in primary care and its effect on health care utilization and cost. Fam Pract 2011;28:516-23.

4 Barnett K, Mercer SW, Norbury M, et al. Epidemiology of multimorbidity and implications for health care, research, and medical education: a cross-sectional study. Lancet 2012;380:37-43.

5 Wallace E, Salisbury C, Guthrie B, et al. Managing patients with multimorbidity in primary care. BMJ 2015;350:h176.

6 Wallace E, McDowell R, Bennett K, et al. Comparison of count-based multimorbidity measures in predicting emergency admission and functional decline in older community-dwelling adults: a prospective cohort study. BMJ Open 2016;6:e013089.

7 Lawson KD, Mercer SW, Wyke S, et al. Double trouble: the impact of multimorbidity and deprivation on preference-weighted health related quality of life a cross sectional analysis of the Scottish health survey. Int J Equity Health 2013;12:67.

8 Bayliss EA, Ellis JL, Steiner JF. Barriers to self-management and quality-of-life outcomes in seniors with multimorbidities. Ann Fam Med 2007:5:395-402.

9 Bratzke LC, Muehrer RJ, Kehl KA, et al. Self-Management priority setting and decision-making in adults with multimorbidity: a narrative review of literature. Int J Nurs Stud 2015;52:744-55.

10 Coventry PA, Small N, Panagioti M, et al. Living with complexity; marshalling resources: a systematic review and qualitative metasynthesis of lived experience of mental and physical multimorbidity. BMC Fam Pract 2015;16:171.

11 Fortin M, Bravo G, Hudon C, et al. Psychological distress and multimorbidity in primary care. Ann Fam Med 2006;4:417-22.

12 Olaya B, Domènech-Abella J, Moneta MV, et al. All-cause mortality and multimorbidity in older adults: the role of social support and loneliness. Exp Gerontol 2017;99:120-6.

13 Peters M, Potter CM, Kelly L, et al. Self-efficacy and health-related quality of life: a cross-sectional study of primary care patients with multi-morbidity. Health Qual Life Outcomes 2019;17:37.

14 Smith SM, Wallace E, O'Dowd T, et al. Interventions for improving outcomes in patients with multimorbidity in primary care and community settings. Cochrane Database Syst Rev 2016;9:1-117.

15 Polley M. Making sense of social prescribing. London: University of Westminster, 2017

16 HSE. Social prescribing, 2019. Available: https://www.hse.ie/eng/ health/hl/selfmanagement/donegal/programmes-services/socialprescribing/social-prescribing.html

17 HSE. Waterford social prescribing service: Waterford libraries, 2018. Available: http://waterfordlibraries.ie/waterford-social-prescribingservice/

18 Bickerdike L, Booth A, Wilson PM, et al. Social prescribing: less rhetoric and more reality. A systematic review of the evidence. BMJ Open 2017;7:e013384.

19 Mercer SW, Fitzpatrick B, Grant L, et al. Effectiveness of communityLinks practitioners in areas of high socioeconomic deprivation. Ann Fam Med 2019;17:518-25.

20 Smith S, O'Donnell P. Deep end Ireland, 2018. http://deepend.ie/wpcontent/uploads/2018/04/Deep-End-Ireland-Link-Workers-Report2018.pdf. Available: http://deepend.ie/ [Accessed 21/01/2021].

21 Chan A-W, Tetzlaff JM, Altman DG, et al. Spirit 2013 statement: defining standard protocol items for clinical trials. Ann Intern Med 2013;158:200-7.

22 Moher D, Hopewell S, Schulz KF, et al. Consort 2010 explanation and elaboration: updated guidelines for reporting parallel group randomised trials. BMJ 2010;340:c869. 
23 HIQA. Guidelines for the economic evaluation of health technologies in Ireland, 2020. https://www.hiqa.ie/sites/default/files/2020-09/ HTA-Economic-Guidelines-2020.pdf. Available: https://www.hiqa.ie [Accessed 21/01/2021].

24 Craig P, Dieppe P, Macintyre S, et al. Developing and evaluating complex interventions: the new medical Research Council guidance. BMJ 2008;337:a1655.

25 Moore GF, Audrey S, Barker M, et al. Process evaluation of complex interventions: medical Research Council guidance. BMJ 2015;350:h1258.

26 Haase T. The 2016 Pobal HP deprivation index (SA) trutzhaase. eu2016, 2016. Available: http://trutzhaase.eu/deprivation-index/the2016-pobal-hp-deprivation-index-for-small-areas/

27 Ireland DE. Deep end Ireland, 2020. Available: www.deepend.ie

28 McCarthy C, Clyne B, Corrigan D, et al. Supporting prescribing in older people with multimorbidity and significant polypharmacy in primary care (SPPiRE): a cluster randomised controlled trial protocol and pilot. Implement Sci 2017;12:99.

29 OECD. Skills matter: further results from the survey of adult skills, OECD skills studies. Paris: OECD Publishing, 2016.

30 Mercer SW, Fitzpatrick B, Grant L, et al. The Glasgow 'Deep End' Links Worker Study Protocol: a quasi-experimental evaluation of a social prescribing intervention for patients with complex needs in areas of high socioeconomic deprivation. J Comorb 2017;7:1-10.

31 Lovell R, Husk K, Blockley K, et al. A realist review and collaborative development of what works in the social prescribing process. The Lancet 2017;390:S62.

32 Alliance. Record of learning; defining the links approach, 2017. Available: https://www.alliance-scotland.org.uk/wp-content/uploads/ 2017/11/Defining-the-Links-Approach-Module-Final.pdf

33 Langford CP, Bowsher J, Maloney JP, et al. Social support: a conceptual analysis. J Adv Nurs 1997;25:95-100.

34 Smith SM, Wallace E, Salisbury C, et al. A core outcome set for multimorbidity research (COSmm). Ann Fam Med 2018;16:132-8.

35 Al-Janabi H, Flynn TN, Coast J. Development of a self-report measure of capability wellbeing for adults: the ICECAP-A. Qual Life Res 2012;21:167-76.
36 Gusi N, Olivares PR, Rajendram R. The EQ-5D health-related quality of life questionnaire. In: Preedy VR, Watson RR, eds. Handbook of disease burdens and quality of life measures. New York, NY: Springer New York, 2010: 87-99.

37 Brennan C, Worrall-Davies A, McMillan D, et al. The hospital anxiety and depression scale: a diagnostic meta-analysis of case-finding ability. J Psychosom Res 2010;69:371-8.

38 Schuling J, de Haan R, Limburg M, et al. The Frenchay activities index. assessment of functional status in stroke patients. Stroke 1993;24:1173-7.

39 Hibbard JH, Stockard J, Mahoney ER, et al. Development of the patient activation measure (PAM): conceptualizing and measuring activation in patients and consumers. Health Serv Res 2004;39:1005-26.

40 Duncan P, Murphy M, Man M-S, et al. Development and validation of the multimorbidity treatment burden questionnaire (MTBQ). BMJ Open 2018;8.

41 StataCorp. Stata statistical software: 15 ed. College Station, TX: StataCorp LLC, 2017.

42 HSE. Evaluation report of the Donegal social prescribing service, 2014. Available: https://www.hse.ie/eng/services/list/4/mentalhealth-services/nosp/research/reports/donegal-social-prescribingevaluation.pdf

43 Gobeil-Lavoie A-P, Chouinard M-C, Danish A, et al. Characteristics of self-management among patients with complex health needs: a thematic analysis review. BMJ Open 2019;9:e028344.

44 NHS. NHS long term plan: NHS, 2019. Available: https://www. longtermplan.nhs.uk

45 Oireachtas Hot. Committee on the future of healthcare. Sláintecare report Ireland, 2017. Available: https://www.gov.ie/en/publication/ 0d2d60-slaintecare-publications/\#the-slaintecare-report

46 RialtasnahEireann. Sláintecare implementation strategy, 2018. Available: https://www.gov.ie/en/publication/0d2d60-slaintecarepublications/\#the-slaintecare-report

47 Carnes D, Sohanpal R, Frostick C, et al. The impact of a social prescribing service on patients in primary care: a mixed methods evaluation. BMC Health Serv Res 2017;17:1-9. 